\title{
Intervención y puesta en valor de la Torre del Molinete en Mazarrón y su entorno paisajístico y patrimonial Pedro E. Collado Espejo
}

Universidad Politécnica de Cartagena, Cartagena, España, pedroe.collado@upct.es

\begin{abstract}
The Tower of El Molinete is one of the three watchtowers built between the 15th and 16th centuries in Mazarron (Murcia - Spain), in order to warn the population of eventual incursions by Berber pirates. The Tower is located in the urban area of Mazarron and it crowns the so-called Hill of El Molinete, which is the great green area of the city. Both the Tower and the Hill were in a poor state of preservation. The intervention has resulted in the improvement of the state of preservation and accessibility to the Tower, as well as the regeneration, fitting-out and landscaping of the entire Hill, as an open and safe urban space recovered for leisure and enjoyment of the historical and natural environment, by reintegrating these two heritage and landscape points of reference into the city.
\end{abstract}

Key words: intervention, tower, hill, accessibility.

\section{Introducción}

La Torre del Molinete es una de las tres torres defensivas costeras, construidas entre los siglos XV y XVI, que se encuentran en el municipio de Mazarrón (Murcia, España), las otras dos son la Torre de Santa Isabel o de Las Cumbres y la Torre de Los Caballos. Estas tres torres vigía, declaradas Bien de Interés Cultural con categoría de Monumento (con la entrada en vigor de la Ley 16/1985 de Patrimonio Histórico Español), se construyeron como consecuencia de la alta frecuencia con que se producían, en aquella época, las incursiones de piratas berberiscos en todo el litoral murciano $\mathrm{y}$, especialmente, en la costa de Mazarrón. La Torre del Molinete es la única de las tres torres que se encuentran en el casco urbano de Mazarrón, pues las otras están en la línea de costa, y corona el llamado Cerro del Molinete, que es la gran zona verde del núcleo urbano de la ciudad, dominando así toda la bahía como puesto de vigilancia y aviso a la población frente a la posible llegada de invasores desde el mar.
La intervención y puesta en valor de la Torre del Molinete, en Mazarrón, y su entorno paisajístico y patrimonial se engloba dentro de las actuaciones previstas en el "Plan Director de Infraestructuras, Accesibilidad y Equipamientos Turísticos de la Bahía de Mazarrón”, aprobado en 2003, y que marcaba como uno de los principales objetivos para los años siguientes la planificación de las actuaciones a desarrollar para la mejora, en todos los ámbitos, de la Bahía de Mazarrón con el fin de convertirla en un referente cultural y turístico de la Región de Murcia, prestando especial atención a todos los recursos históricos, sociales, arquitectónicos, naturales, paisajísticos y culturales que posee esta zona y a su potencialidad. En concreto, este Plan Director, en el apartado correspondiente al análisis y propuestas de actuación en los "Recursos Históricos y Culturales", detalla unos criterios y metodología de intervención que se han seguido en la Torre y Cerro del Molinete, tanto en la fase de proyecto como en la propia ejecución de las actuaciones, con el fin de 
recuperar, poner en valor y potenciar la Torre como nuevo recurso turístico-cultural a la vez que conseguir la regeneración y mejora del Cerro como espacio natural y paisajístico. Y todo ello prestando especial atención a la restauración de la Torre como monumento, la mejora de las condiciones de seguridad y accesibilidad a la construcción histórica, el acondicionamiento del entorno natural y paisajístico del Cerro como espacio público de esparcimiento y disfrute de la población y visitantes, y la delimitación, vallado y protección de todo el entorno patrimonial para su correcto mantenimiento y conservación.

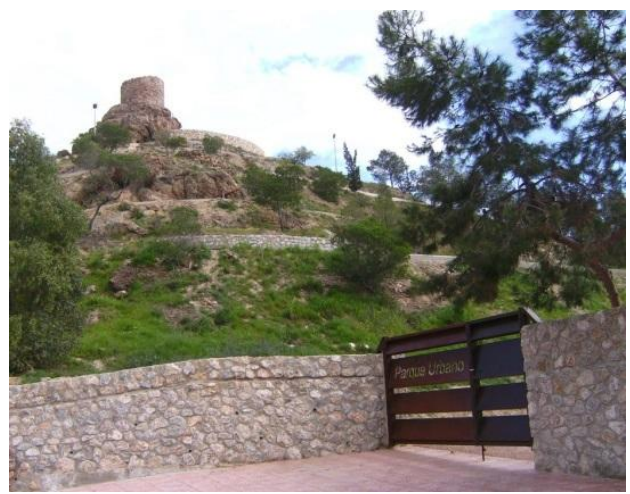

Fig. 1.- Torre y Cerro del Molinete después de la intervención. (P.E. Collado)

\section{Breve descripción de la Torre y el Cerro}

La Torre del Molinete es una construcción de carácter defensivo, realizada en mampostería de piedra caliza tomada con argamasa de cal y de planta circular, por lo que parece que recibe el nombre de "El Molinete" por su gran parecido con la tipología de los molinos de viento, tan presentes en estos entornos. Al estar edificada en la parte más alta del Cerro del Molinete, dispone de unas privilegiadas vistas sobre todo su entorno, con visión directa a las torres de vigilancia situadas en primera línea de la costa de la Bahía de Mazarrón, es decir, las torres de Santa Isabel, Los Caballos, Cope (municipio de Águilas) y Santa Elena (municipio de Cartagena) sirviendo claramente como importante bastión de vigilancia y defensa de Mazarrón. Por su situación elevada sobre unas rocas, en la cima del cerro, antes de la intervención carecía de un acceso fácil y su interior disponía de una única sala, con unas dimensiones bastante reducidas (la Torre tiene 21,20 $\mathrm{m}^{2}$ de superficie construida y un diámetro interior de 2,50 m aprox.), lo que corresponde a una construcción defensiva y pensada únicamente como puesto de vigilancia. La torre presentaba un estado de conservación bastante lamentable ya que no había sido objeto de actuación alguna de reparación o restauración al menos en las últimas décadas. La construcción no presentaba ningún elemento de cubierta, había perdido parte del volumen superior y no tenía ningún elemento de protección al estar abierto el hueco original de la puerta de acceso (posiblemente de madera en origen pero que no se había conservado).

El Cerro del Molinete se ubica en una zona libre y pública del municipio, abierta y $\sin$ ningún tipo de cerramiento que permita regular y controlar el acceso al cerro, actuando como una gran zona verde dentro del casco urbano. El Cerro tiene una superficie de unos $19.275 \mathrm{~m}^{2}$ y $935,00 \mathrm{ml}$ de caminos, que discurren por las laderas del monte para conducir a la cima, donde se encuentra la Torre. Por su elevación, cuenta con importantes vistas sobre el entorno, destacándose como un importante punto de observación de los edificios históricos referentes de Mazarrón, como son las Casas Consistoriales, el Castillo de los Vélez, las Iglesias de San Antonio de Padua, de San Andrés y de La Purísima, el Coto Minero de San Cristóbal.

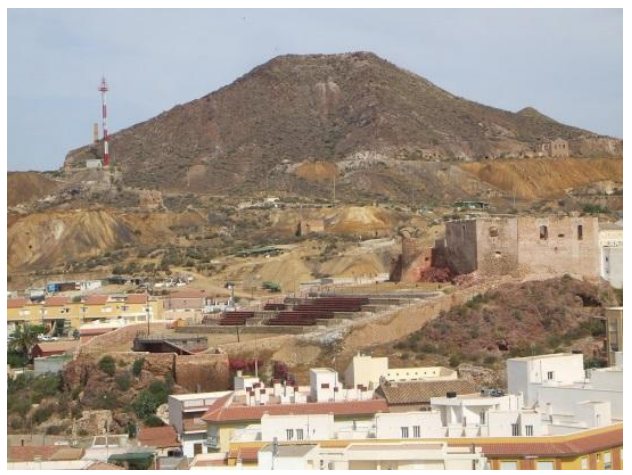

Fig. 2.- Vista general del Castillo de los Vélez y el Coto Minero de San Cristóbal desde la Torre del Molinete. (P.E. Collado) 
En cuanto al estado de conservación del Cerro, podemos decir que la apariencia era de un entorno natural semiabandonado, donde destacaba la proliferación de arbustos y matorrales entre el numeroso arbolado y con los caminos de tierra que conducían a la cima pareciendo abrirse paso entre la maleza. La falta de cercado y protección había ocasionado un cierto deterioro del entorno, ya que no permitía controlar el depósito de basuras y escombros, favoreciendo además los actos vandálicos, con rotura de farolas, pintadas en la Torre, rotura de varios bancos...

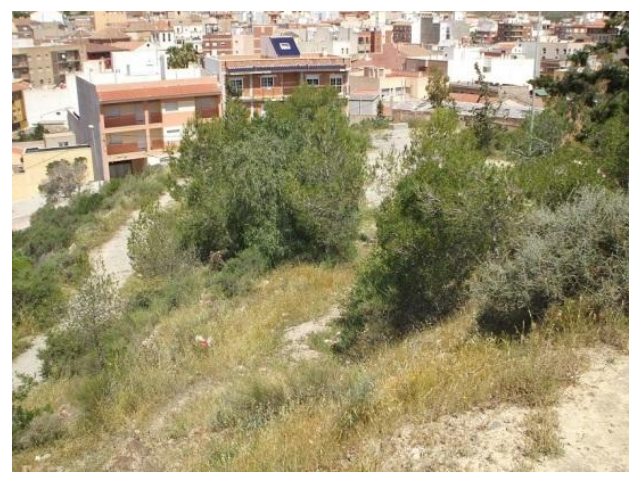

Fig. 3.- Detalle de la ladera del Cerro antes de la intervención. (P.E. Collado)

\section{Intervención en la Torre}

La intervención proyectada y realizada en la Torre se planteó con tres objetivos básicos. En primer lugar, se debía consolidar y restaurar la edificación, pero teniendo muy en cuenta que se interviene en un Bien de Interés Cultural con categoría de Monumento; por tanto, la metodología de conocimiento y los criterios de actuación debían ser absolutamente respetuosos con los valores históricos, arquitectónicos, sociales y culturales que atesora la Torre. La restauración se limitó a procesos básicos de limpieza de los paramentos existentes, consolidación y conservación de los muros de mampostería intentando, en todo momento, diferenciar (aunque de manera sutil para no distorsionar la imagen general del monumento), los morteros y materiales pétreos nuevos a colocar respecto de los originales, para evitar caer en el llamado falso histórico.

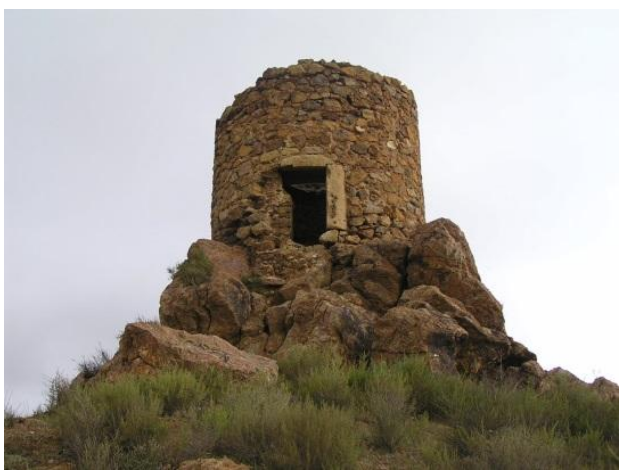

Fig. 4.- Imagen general de la Torre del Molinete antes de la restauración. (P.E. Collado)

En segundo lugar, se buscaba una mejora de las condiciones de accesibilidad y seguridad al inmueble. En este caso, a pesar de ser un objetivo derivado del cumplimiento de la Ley Regional de Accesibilidad, por las propias características constructivas y de ubicación de la Torre, no resultaba posible garantizar la accesibilidad total al interior de la construcción sin alterar gravemente su configuración arquitectónica original (protegida por su carácter de Bien de Interés Cultural). No obstante, se proyectaron y realizaron una serie de acciones encaminadas a garantizar el acceso de todas las personas al menos a un punto lo más cercano posible a la base de la Torre y, en cualquier caso, a la nueva plataforma de descanso y recreo, a los pies de la Torre, donde se instalarían finalmente los carteles y puntos de información e interpretación de toda la intervención.

En tercer lugar, se buscaba el acondicionamiento natural y paisajístico del entorno inmediato de la edificación histórica como espacio público al objeto de mejorar su imagen y sus condiciones como lugar de acogida de visitantes. Sobre este nuevo espacio (una explanada delante del acceso principal de la Torre) se han centrado las actuaciones para la mejora de accesibilidad y las acciones informativas, pero además, en los recuperados caminos del Cerro se han diseñado y creado pequeñas zonas de estancia y descanso para los visitantes, con un mobiliario urbano (bancos, papeleras, cartelería...) diseñados acorde con el entorno histórico, natural y paisajístico del Molinete. 
Se procedió a la limpieza general de la Torre y su entorno inmediato; con eliminación de las pintadas existentes con el uso de proyección de agua destilada a presión controlada y cepillado manual con cepillo de celdas suaves, además de aplicar un tratamiento hidrofugante como protección final (probado previamente para que no afectase a la textura y color del cerramiento original de mampostería). Las pequeñas grietas fueron inyectadas con lechada de cal y cosidas con varillas de fibra de vidrio, consiguiendo la continuidad y estabilidad estructural de la fábrica. En zonas con lagunas y carencias de material, se realizó la reintegración con material pétreo de distinta tonalidad de la existente, aunque sin destacarse en exceso, y mortero de cal coloreado para su entonación con el mortero existente, en una intervención que perseguía la diferenciación sutil entre materiales originales y nuevos pero con un marcando interés de recomposición integradora. Para el remate superior de la fábrica de mampostería se optó por la consolidación mediante aplicación de mortero de cal pero tomando la forma ligeramente curva para la correcta evacuación del agua de lluvia. Con esta solución se consolidaba la fábrica afectando mínimamente al volumen existente, evitando la restitución con material nuevo.

La puerta de acceso original, posiblemente de madera, no se había conservado ni había constancia gráfica o escrita de cómo fue en origen. Además, en el hueco de acceso faltaba una de las jambas de piedra caliza por lo que se decidió la reposición de ésta pero realizándola en piedra artificial para conseguir cierta diferenciación visual respecto de la original. Para cerrar y proteger el acceso al interior de la Torre se colocó una puerta de forja, a modo de enrejado, que permite ver el interior sin acceder a él. Finalmente, a toda la fábrica se le daría un tratamiento protector e hidrofugante que no producía brillos en los paramentos.

Para salvar la importante diferencia de cota existente entre explanada y el hueco de acceso a la Torre, así como la presencia de las rocas, se optó por realizar una nueva escalera con peldañeado de hormigón coloreado, en tonos ligeramente almagra, para conseguir la máxima integración cromática de esta solución con las enormes rocas sobre las que descansa la Torre y el menor impacto visual posible. Para una mayor seguridad en la subida, se colocó una liviana barandilla metálica que se integra perfectamente en todo el conjunto al haberse tratado con una imprimación en tonos similares a los de las rocas del entorno.

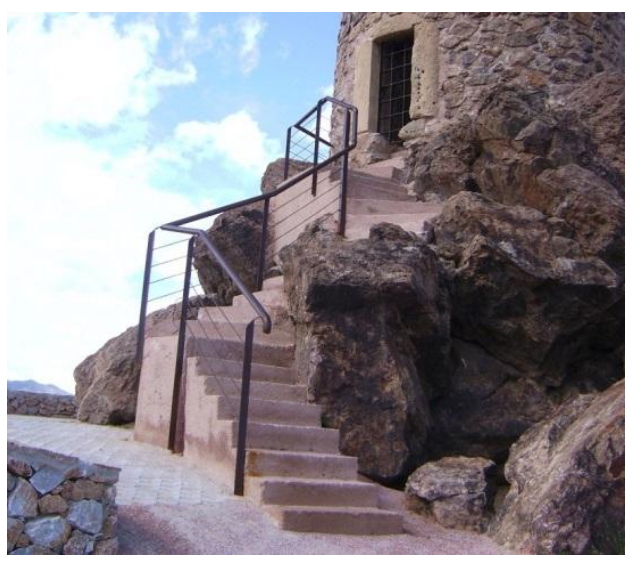

Fig. 5.- Detalle de la nueva escalera de hormigón coloreado y barandilla metálica para acceso a la Torre. (P.E. Collado)

El interior de la Torre presentaba un pavimento de tierra natural, muy compacta, del que sobresalía ligeramente la base rocosa sobre la que asienta la construcción. Después de varias pruebas, se decidió colocar un relleno de piedras de canto rodado del color semiblanco que presentaba la zona con tierra natural, hasta el nivel de la roca pero quedando ésta vista para no modificar la cota interior del pavimento.

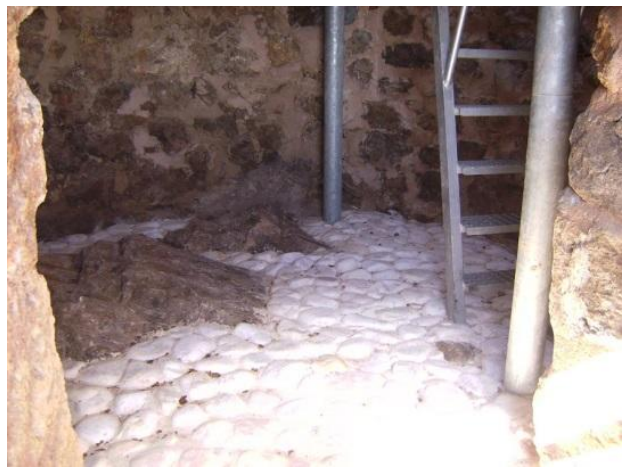

Fig. 6.- Detalle del nuevo pavimento de canto rodado del interior de la Torre. (P.E. Collado) 
Para la nueva cubierta se optó por una plataforma, a modo de mirador, con tarima de madera de pino cuperizado apoyada en una estructura metálica ligera sobre tres pilares redondos y de acero. Esta plataforma se sitúa por encima del arranque de la bóveda interior, que estaba derruida y no se quiso reconstruir, de tal forma que los propios muros de la torre hacen de peto de protección y así el visitante disfruta de una posición elevada sobre la que poder contemplar el casco urbano de Mazarrón. Para el acceso a la cubierta se colocó una escalera de acero inoxidable y con una fuerte pendiente, a modo casi de escala, por la estrechez interior y la altura a salvar.

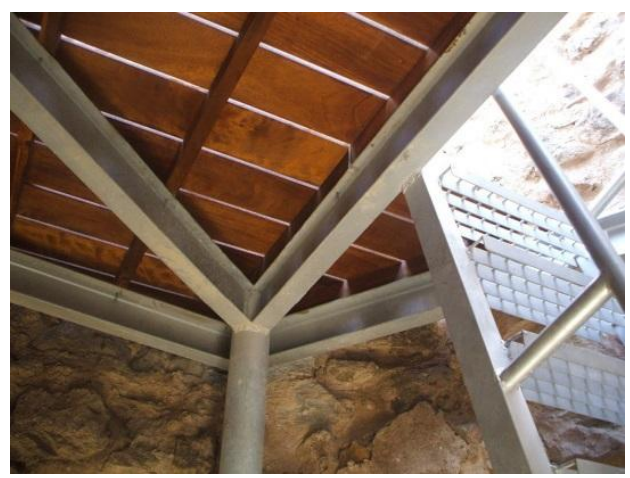

Fig. 7.- Detalle del interior de la Torre con la solución de la cubierta. (P.E. Collado)

Por último, para facilitar el acceso a la explanada natural que daba a la Torre (tramo final de la senda que recorre el Cerro), había que salvar una pendiente muy pronunciada del camino por lo que se realizó una rampa escalonada mediante traviesas de tren y balsas de albero compactado y estabilizado, ejecutando un murete de mampostería, con piedras similares a las existentes en el Cerro, para contención de tierras y delimitación del nuevo entorno nivelado, coronado por una ligera barandilla metálica para mayor seguridad y facilidad de acceso. La explanada natural se transformó así en una pequeña plataforma para estancia y reposo del visitante y donde se pueden realizar acciones informativas. Esta plataforma se pavimentó con losa calada de hormigón vibromoldeado y con tratamiento verde entre resaltes a base de plantación de césped.

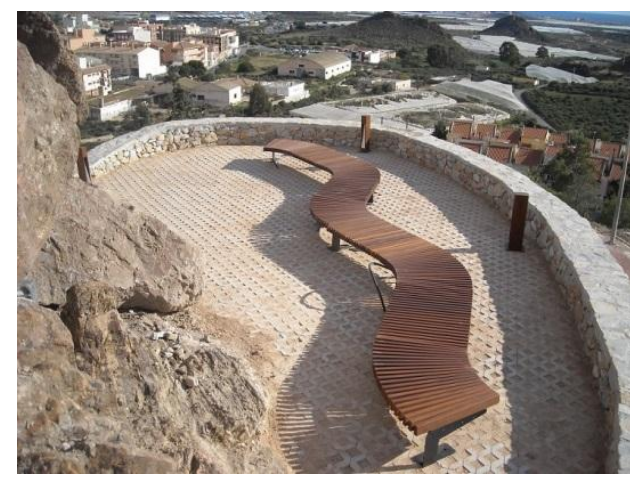

Fig. 8.- Plataforma para estancia y reposo del visitante frente a la Torre. (P.E. Collado)

Como mobiliario urbano para esta plataforma se colocó un banco curvo de madera, con un diseño moderno (sin respaldo y formando una "s" en planta), una papelera a juego con el banco y unas luminarias, tipo balizas y antivandálicas, realizadas en fundición de acero, de una altura de $0,50 \mathrm{~m}$ y diseño exclusivo para integrarlas en este entorno turístico-cultural. Para completar esta intervención se ha colocado finalmente un cartel informativo sobre las características de la Torre y su significado histórico y cultural.

\section{Intervención en el Cerro}

El Cerro del Molinete tiene un importante valor paisajístico pues sobresale especialmente sobre la silueta del conjunto urbano de Mazarrón, siendo visible desde prácticamente todos los puntos de la ciudad y es, como se ha comentado, un perfecto observatorio del casco urbano desde una cota elevada, pues permite la identificación y observación de casi todos los monumentos de Mazarrón. Por tanto, la intervención se planteó con tres objetivos: mejora del entorno ambiental y paisajístico, mejora de accesos y caminos y cierre perimetral del recinto. En primer lugar se procedió a la limpieza y retirada de escombros y basuras así como la poda y desbroces parciales en algunas zonas, para permitir la mejora y ejecución de caminos, nuevos cerramientos e instalaciones. En cuanto al mobiliario existente, se reparó y se puso en uso la fuente, se limpiaron de pintadas los bancos de hormigón y se reubicaron éstos para mejorar su uso y para permitir la ejecución de los nuevos cerramientos 
y caminos. Para hacer más atractivo y relajante el paseo por los caminos del Cerro se habilitaron tres zonas de estancia y descanso. La primera en una de las pocas plataformas horizontales con que ya contaba el Cerro, realizándose muros de mampostería en el borde interior del recinto para protección y descanso del visitante. La segunda, a mitad de la subida al Cerro. Por último, se habilitó una tercera plataforma abierta, de forma circular, en el cruce de caminos que se produce a media subida del Cerro, creando una pequeña zona de encuentro y descanso.

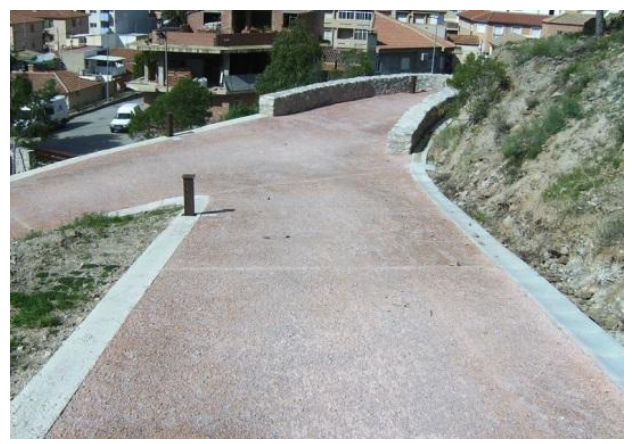

Fig.9.- Detalle de camino terminado con zona encuentro y descanso al fondo. (P.E. Collado)

Estas zonas se han realizado con muretes de mampostería de unos $65 \mathrm{~cm}$ de altura y un pavimento a base de solera-cemento de $15 \mathrm{~cm}$ de espesor, formada por árido lavado de $10 \mathrm{~mm}$, con mezcla pobre de cemento, colorante mineral y fibra de vidrio, además de un acabado algo rugoso conseguido con el compactado mecánico con rulo. Además, para iluminar estas zonas se colocaron las mismas balizas de fundición que se diseñaron y colocaron en la plataforma al pié de la Torre.

El Cerro tiene unos 935 metros de caminos que permiten la subida en espiral hasta la cima, donde se encuentra la Torre. Estos caminos eran de tierra, pero estaban bastante deteriorados pues se producían desprendimientos desde la ladera y arrastres de tierras, además de la presencia de malas hierbas y vegetación silvestre y la formación de numerosos charcos de agua cuando llovía, impidiendo el normal uso y disfrute de este espacio verde. Por tanto, la intervención debía mejorar las condiciones de tránsito de las personas (y ocasionalmente de los vehículos de mantenimiento y conservación) y el acceso a la Torre, independientemente de las condiciones meteorológicas.

Se realizó una limpieza general de caminos, con eliminación de piedras, desbroce de vegetación y raíces y una ligera corrección de las pendientes de los caminos y plataformas a tratar, con especial eliminación de taludes poco estables, suavizando su pendiente, al objeto de evitar futuros desprendimientos. Para estabilizar y marcar los caminos, con una anchura media de unos 3 metros, se procedió a la ejecución de una correa de hormigón de borde exterior y unas canaletas prefabricadas de hormigón para la recogida de pluviales, al interior, y situadas cada $10 \mathrm{~m}$ bajo un canal de drenaje, con una rejilla registrable de fundición en la cara superior. Al igual que con las tres zonas estanciales y de descanso, el pavimento de los caminos se hizo a base de solera-cemento, con mezcla pobre de cemento, colorante mineral y fibra de vidrio, además del acabado algo rugoso. La iluminación se resolvió con las balizas de fundición ya comentadas, quedando así todas las zonas de paso con el mismo acabado y sistema de iluminación.

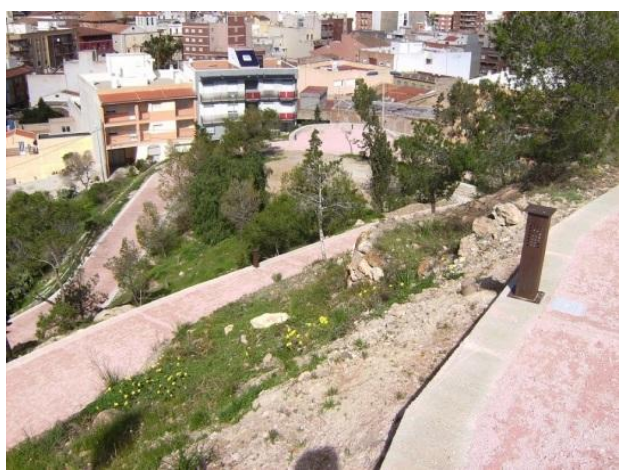

Fig.10.- Detalle de la ladera del Cerro después de la intervención. (P.E. Collado)

Como se ha comentado, a los problemas de falta de mantenimiento y conservación que tenía el Cerro se añadía la ausencia de un correcto cercado, lo que había favorecido el estado de semiabandono que presentaba todo el entorno, pues esta situación no permitía controlar el depósito de basuras e impedir la acumulación de escombros y los numerosos actos vandálicos que 
se apreciaban en el mobiliario urbano. Por tanto, una de las actuaciones más importantes para la correcta conservación del Cerro del Molinete ha consistido en la ejecución de un cerramiento completo del recinto del Cerro a base de muros de mampostería realizados con piedras de las mismas características de las existentes en el propio Cerro y una malla superior de acero electrosoldado, colocando dos puertas de acceso al entorno vallado en las dos zonas originales de entrada al recinto del Cerro y donde comienzan los dos caminos hacia la Torre.

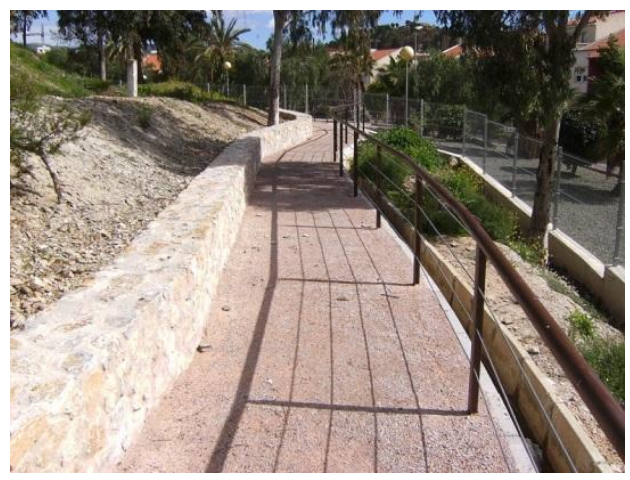

Fig.11.- Detalle de camino terminado con vallado del recinto al fondo. (P.E. Collado)

La altura de los nuevos muros de mampostería dependió de la zona sobre la que se actuaba y de si tenían que contener tierras o no. En el perímetro de arranque del Cerro existían tramos de muro de mampostería que fue necesario limpiar de pintadas y reparar las pequeñas grietas y faltas de material. La altura media que presentaban estos muros de mampostería era de unos 1,50 metros (alguno llegaba a los 1,90 m), por lo que se decidió mantener la altura y colocar sobre ellos un enrejado de malla de acero electrosoldada. En las zonas donde no había ningún tipo de muro se colocó la misma malla pero con una altura total de unos $3,00 \mathrm{~m}$.

Para el acceso al recinto vallado se diseñaron y colocaron dos puertas de acero corten con la leyenda "parque urbano" para identificar la actuación. Los dos accesos contaban con dos puertas independientes, una para el acceso peatonal (de 1,00 m de anchura), y la otra con un ancho de 3,60 m para el acceso de los vehículos de mantenimiento y conservación.

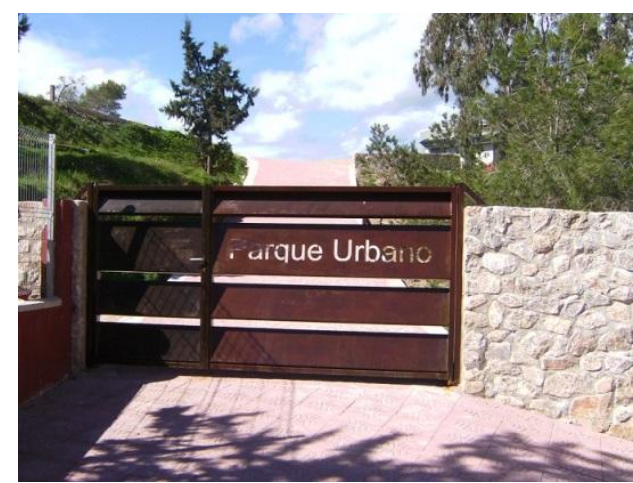

Fig.12.- Detalle de una de las dos puertas de acceso al recinto del Cerro. (P.E. Collado)

Por último, la actuación se centró en la mejora ambiental y paisajística del Cerro del Molinete. Para ello se recurrió a la plantación, en las zonas donde se había perdido vegetación, de especies vegetales autóctonas y de bajo consumo de agua, garantizando así perfecta adaptación al entorno, Se trataba de crear un verdadero parque urbano, una zona verde, con las características del monte natural del municipio de Mazarrón, añadiendo información sobre las diferentes especies vegetales presentes y las características de los hábitat creados.

\section{Conclusiones}

Las actuaciones desarrolladas en la Torre y Cerro del Molinete en Mazarrón han supuesto, en primer lugar, la restauración y puesta en valor de la antigua Torre vigía como un importante patrimonio histórico, arquitectónico, cultural y monumental de la ciudad. Teniendo en cuenta en todo momento que la Torre es un Bien de Interés Cultural con categoría de Monumento, se ha limpiado y consolidado la volumetría existente y las estructuras originales de muros de mampostería con el empleo de materiales similares y técnicas constructivas tradicionales, recuperando el acceso original al interior de la edificación con la ejecución de una escalera que se integra en el entorno y permite el acceso seguro al inmueble y, con la ejecución de la nueva cubierta transitable, recuperando la función original para la que fue construida, es decir, servir de vigilancia-mirador del casco urbano y de la Bahía de Mazarrón. 
Por tanto, la intervención llevada a cabo en la Torre del Molinete ha permitido la recuperación y puesta en valor de un importante referente arquitectónico y patrimonial de Mazarrón.

En cuanto al Cerro sobre el que se eleva la Torre del Molinete, la intervención realizada ha conseguido su regeneración como espacio natural y de esparcimiento así como su recuperación paisajística para el disfrute de la población y de los visitantes.

Destacar que la mejora de las condiciones de conservación y accesibilidad a la Torre así como el acondicionamiento y ajardinamiento integral del Cerro como espacio urbano abierto y seguro, recuperado para el esparcimiento y disfrute de los ciudadanos y visitantes de Mazarrón, como un entorno histórico y natural, con sus caminos, la iluminación ornamental, el nuevo mobiliario urbano y los varios paneles informativos $\mathrm{y}$ divulgativos que se han colocado, han hecho posible la revitalización e integración definitiva de estos dos importantes referentes culturales, patrimoniales y paisajísticos en el día a día de Mazarrón.

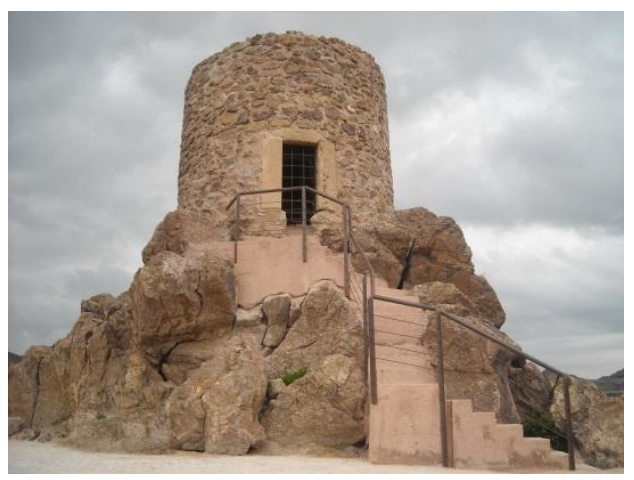

Fig. 13.- Imagen de la Torre del Molinete después de la restauración. (P.E. Collado)

\section{Ficha técnica de la intervención}

Promotor/Propiedad: Consorcio Turístico de Mazarrón. Gerente: Pío Garrido Urbano.

Proyecto/Dirección de Obra: Rafael Pardo Prefasi, Severino Sánchez Sicilia, Inmaculada González Balibrea y Pedro-E. Collado Espejo. Empresa de restauración: Ingeniería Quipons SL Presupuestos de licitación: Torre, 87.052,83 € Cerro, $175.038,10 €$

\section{Referencias}

Alonso S. (1990). Libro de los castillos y fortalezas de la Región de Murcia. Asociación Nacional de Amigos de los Castillos. Murcia.

Collado P.E., González I., Pardo R., Sánzhez S. (2011). “Torre y Cerro del Molinete en Mazarrón: intervención en el patrimonio arquitectónico y paisajístico para su puesta en valor como recurso turístico y cultural", en XXII Jornadas de Patrimonio Cultural de la Región de Murcia. Ediciones Tres Fronteras. Murcia, pp. 223-233.

Munuera D. (2006). "Una historia singular: la costa del Reino de Murcia y el nacimiento de Mazarrón durante la etapa fronteriza (siglos XIII-XVI)", en El Siglo del Milagro. Casas y Villa de los Alumbres de Almazarrón. Ligia Comunicación SL. Ayuntamiento de Mazarrón. pp. 22-39.

Pardo R., Sánchez S. (2003). Plan Director de Infraestructuras, Accesibilidad y Equipamientos Turísticos de la Bahía de Mazarrón. Ayuntamiento de Mazarrón. 